\title{
Accommodation of the spinal cat to a tripping perturbation
}

\section{Hui Zhong ${ }^{1}$, Roland R. Roy ${ }^{1,5}$, Kenneth K. Nakada ${ }^{1}$, Sharon Zdunowski ${ }^{1}$, Nicole Khalili ${ }^{1}$, Ray D. de Leon ${ }^{3}$ and V. Reggie Edgerton $1,2,4,5 *$}

1 Departments of Integrative Biology and Physiology, University of California Los Angeles, Los Angeles, CA, USA

${ }^{2}$ Department of Neurobiology, University of California Los Angeles, Los Angeles, CA, USA

${ }^{3}$ Kinesiology and Nutritional Science, California State University Los Angeles, Los Angeles, CA, USA

${ }^{4}$ Department of Neurosurgery, University of California Los Angeles, Los Angeles, CA, USA

${ }^{5}$ Brain Research Institute, University of California Los Angeles, Los Angeles, CA, USA

\section{Edited by:}

Alexander Rabchevsky, University of Kentucky, USA

Reviewed by:

Christy Carter, University of Florida USA

Vaughan G. Macefield, University of Western Sydney, Australia

*Correspondence:

V. Reggie Edgerton, Department of Integrative Biology and Physiology, University of California Los Angeles, 610 Charles E. Young Drive East, Los Angeles, CA 90095-7239, USA

e-mail:vre@ucla.edu
Adult cats with a complete spinal cord transection at T12-T13 can relearn over a period of days-to-weeks how to generate full weight-bearing stepping on a treadmill or standing ability if trained specifically for that task. In the present study, we assessed short-term (milliseconds to minutes) adaptations by repetitively imposing a mechanical perturbation on the hindlimb of chronic spinal cats by placing a rod in the path of the leg during the swing phase to trigger a tripping response. The kinematics and EMG were recorded during control (10 steps), trip (1-60 steps with various patterns), and then release (without any tripping stimulus, 10-20 steps) sequences. Our data show that the muscle activation patterns and kinematics of the hindlimb in the step cycle immediately following the initial trip (mechanosensory stimulation of the dorsal surface of the paw) was modified in a way that increased the probability of avoiding the obstacle in the subsequent step. This indicates that the spinal sensorimotor circuitry reprogrammed the trajectory of the swing following a perturbation prior to the initiation of the swing phase of the subsequent step, in effect "attempting" to avoid the re-occurrence of the perturbation. The average height of the release steps was elevated compared to control regardless of the pattern and the length of the trip sequences. In addition, the average impact force on the tripping rod tended to be lower with repeated exposure to the tripping stimulus. EMG recordings suggest that the semitendinosus, a primary knee flexor, was a major contributor to the adaptive tripping response. These results demonstrate that the lumbosacral locomotor circuitry can modulate the activation patterns of the hindlimb motor pools within the time frame of single step in a manner that tends to minimize repeated perturbations. Furthermore, these adaptations remained evident for a number of steps after removal of the mechanosensory stimulation.

Keywords: EMG, spinal locomotor circuits, mechanosensory stimulation, tripping response, spinal cord transection

\section{INTRODUCTION}

While experience-dependent plasticity clearly occurs in supraspinal circuits controlling motor tasks, we now know that experiencedependent plasticity also occurs in spinal circuits that control posture and locomotion. For example, Di Giorgio and Menzio (1946) reported that the asymmetric positions of the lower limbs that are acquired after being exposed to an asymmetric vestibular input for about $30 \mathrm{~min}$ persists after a complete spinal cord transection. Chopin and Buerger (1976) demonstrated that midthoracic, complete spinal rats learned to avoid shock by keeping the paw elevated above a threshold level within 5-20 min of being exposed to a conditioning-learning paradigm. Complete spinal rats also can adapt the hindlimb kinematics to a perturbing force field so that a more normal stepping pattern is achieved within a time frame of seconds to minutes (de Leon et al., 1999; Timoszyk et al., 2002; Heng and de Leon, 2007). Motor learning that occurs over a period of days and weeks has been demonstrated in numerous experiments in which complete spinal animals regain and improve stepping or standing performance with daily practice of these tasks (Barbeau and Rossignol, 1987; Lovely et al., 1990; de Leon et al., 1998a,b). Given that learning-related phenomena can occur within a short time frame (within seconds) presumably within supraspinal circuits (Choi and Bastian, 2007), we hypothesized that a similar learning phenomenon could occur in the spinal cord within a similar time frame. In the present experiments, we demonstrate learning-related responses that occur within seconds of a tripping stimulus applied during the swing phase of a step cycle in the cat after a complete, low-thoracic spinal cord transection. We also found that the spinal circuitry is capable of perceiving a mechanical perturbation of the step cycle in a way that enhances the probability of sustaining successful locomotion, further demonstrating the wide repertoire of sensorimotor processing within the spinal locomotor circuitry.

\section{MATERIALS AND METHODS EXPERIMENTAL DESIGN}

Four adult female cats (2-3 years of age) were used for these studies, with most of the data shown derived from three cats. Prior to any surgery, the cats were acclimated to the treadmill and the testing environment for several sessions, i.e., the cats were made 
to walk bipedally on a treadmill at speeds ranging from 0.2 to $1.0 \mathrm{~m} / \mathrm{s}$ while in a harness supporting the upper body (de Leon et al., 1998a). Intramuscular EMG electrodes were implanted in selected hindlimb muscles and the spinal cord was completely transected at a low-thoracic level. Starting 4 days after surgery, the cats were step trained for $30 \mathrm{~min} /$ day, 5 days/week as described by de Leon et al. (1998a). After 6 months of training, the cats were subjected to tripping experiments periodically as described below. All procedures were performed in accordance with the American Physiological Society Animal Care Guidelines and were approved by the Animal Use Committee at the University of California, Los Angeles.

\section{INTRAMUSCULAR EMG IMPLANTS}

All surgical procedures were performed under aseptic conditions. The cats were administered sodium pentobarbital $(35 \mathrm{mg} / \mathrm{kg}$, i.p.) following pre-treatment with atropine sulfate $(0.05 \mathrm{mg} / \mathrm{kg})$ and acepromazine maleate $(0.2 \mathrm{mg} / \mathrm{kg})$ administered intramuscularly. Supplemental doses of anesthesia were administered as needed to maintain a surgical level. All incisions were closed in layers, i.e., 4.0 chromic gut and 4-0 Ethilon suture were used to suture the fascia and skin, respectively.

Prior to spinal cord transection, intramuscular recording electrodes were implanted in selected hindlimb muscles using procedures described in detail previously (Pierotti et al., 1989; de Leon et al., 1994). Briefly, one amphenol connector having nine Tefloninsulated stainless steel wires (AS 632, Cooner Wire, Chatsworth, CA, USA) was secured to the skull with screws and dental cement. The wires were passed subcutaneously from the connector to the hindlimb. One wire with $\sim 2 \mathrm{~cm}$ of the Teflon removed at the distal end was embedded in the back region and served as a common ground. Two wires were implanted in the following muscles unilaterally: deep mid-region of the distal compartment of the semitendinosus (St), mid-belly of the soleus (Sol), deep portion of the mid-belly of the tibialis anterior (TA), and medial deep portion of the mid-belly of the medial gastrocnemius (MG). The wires were passed through the muscle using a 23-gage hypodermic needle and $\sim 0.5-1.0 \mathrm{~mm}$ of insulation was removed from each wire to form the recording electrodes. After stimulation of the muscle through the connector to ensure the proper placement of the electrodes, each lead was secured with a suture at its entry and exit from the muscle. The proper placement of all electrodes also was verified post-mortem.

\section{SPINAL CORD TRANSECTION}

The spinal cord of each cat was transected completely at $\sim \mathrm{T} 12$ as described in detail previously (Roy et al., 1992). Briefly, a skin incision was made on the back to expose the vertebral processes between $\sim \mathrm{T} 10$ and L1. A partial laminectomy was performed at the T12-T13 vertebral level to expose the spinal cord. Fine scissors and forceps were used to cut the dura longitudinally and to perform a complete transection beginning on the dorsal surface of the cord while preserving the lateral and ventral dura. After the transection, the ends of the cord retracted leaving a clear space. The cut ends of the cord then were lifted gently using fine forceps to assure that no residual spinal cord matter remained between the two cut ends of the cord. Gel foam was packed between the rostral and caudal segments of the cord as an anticoagulant. This procedure allowed for the preservation of the large ventral artery of the spinal cord.

\section{ANIMAL CARE PROCEDURES}

Post-spinal cord transection management of the spinal cats has been detailed elsewhere (Roy et al., 1992). The cats were housed together in spacious cage with the cage floors covered with shredded newspaper. The bladders and colons of the cats were expressed twice daily for the duration of the experiment. Dry kibble and water were given ad libitum and wet food was given once daily.

\section{ANIMAL TESTING}

Prior to the surgeries, the animals were trained to step on the treadmill bipedally. Beginning 7 days post-surgery the animals were step trained for $30 \mathrm{~min} /$ day, 5 days/week for 6 months. All tripping experiments were performed over a period of $\sim 3$ months following the 6-month training period. During this period stepping performance remained stable. During step training, the cats were placed in a thoracic vest that wrapped around the chest and shoulder girdle to provide stability, but allowed the hindlimbs to bear weight and to move freely on the treadmill belt. During the testing while the cats were stepping on the treadmill, an inhouse designed tripping stainless steel rod mounted with a strain gage was placed using a sliding carriage in the normal trajectory of the limb such that it made contact with the dorsal surface of the paw during the swing phase of the step. The tripping rod was mounted onto a sliding chassis that utilized ball bearings to minimize friction and vibration while at the same time maintaining an exact position once in position. This design allowed for a smooth entrance and exit of the rod from the plane of motion of the hindlimb. There was no noise associated with the rod sliding in and out. The chassis was locked into a housing unit that had five different vertical slots allowing the chassis to be moved and locked into five different vertical positions. The results reported below are from the tests when the rod was placed at 1.1 and $3.7 \mathrm{~cm}$ above the treadmill. The base of the housing unit contained slots that allowed horizontal movement of the housing unit itself before it was locked down into position on the treadmill. Thus there was freedom to move the rod horizontally and vertically in the plane of motion before it was locked into place to adjust the system to induce the perturbation at a consistent point during the swing phase of the step cycle. Once the tripping device was set, the rod entered the plane in the same coordinates every time. The strain gage on the rod was calibrated using weights between 10 and $200 \mathrm{~g}$ so that the magnitude of the impact force of the paw onto the rod could be quantified. Each sequence consisted of a series of steps that were either obstructed (trip sequences) or not obstructed (control or release sequences) by the tripping rod during the swing phase of the step cycle. Control steps were either at the beginning of a sequence or at least 50 steps after a series of obstructed steps. Release steps consisted of steps not obstructed that followed immediately after a series of obstructed steps. A typical sequence consisted of a series of control steps (10 steps) followed by an alternating series of trip (range from 1 to 60 ) and release steps (range from 10 to 20 steps). 


\section{DATA RECORDING}

A flexible insulated cable was used to connect the amphenol connector to a recorder. Raw EMG signals were conditioned through a wide band AC differential amplifier at a gain of 1000 and recorded on an analog tape recorder (TEAC Model XR-510, TEAC Corporation, Montebello, CA, USA) with a system bandwidth of D.C. to $2.5 \mathrm{kHz}$. EMG signals were calibrated at the beginning of each recording session using a 1 or $2 \mathrm{mV}$ sine wave $(500 \mathrm{~Hz})$. To record kinematics data, a camera (Panasonic System Camera, WV D5100, Panasonic, Cypress, CA, USA) was oriented perpendicular to the plane of motion. The video was recorded at 30 frames (60 fields) per seconds on VHS tape simultaneously with a signal from a time code generator. The time code generator was used to synchronize the EMG and force recordings. Strain gage force and muscle EMG signals were calibrated, amplified, recorded, and then stored on FM tape using a TEAC recorder. The hindlimbs were shaved and small, round pieces of light-reflecting tape were placed on bony landmarks, i.e., the iliac crest, greater trochanter, head of the tibia, lateral malleolus, base of the calcaneus, head of the fifth metatarsal (MTP), and the first phalanx of the fifth digit, to be used to determine limb segment movement (Figure 1A).

\section{DATA ANALYSIS}

The $x$ and $y$ coordinates of all the bony landmarks were digitized using SIMI Motion and tracked using 2-D tracking within the program. The location of the force bar and the level of the treadmill (zero line) were digitized. The digitized points were exported into text files and then graphed for trajectories and stick diagrams in Excel. Step height was determined by measuring the largest distance between the paw (MTP) and the treadmill belt during the swing phase of the step cycle. Three markers were used to determine changes at each joint angle. Hip angle was based on the iliac crest, greater trochanter, and head of the tibia. Knee angle was based on the greater trochanter, head of the tibia, and lateral malleolus. Ankle angle was based on the head of the tibia, lateral malleolus, and fifth metatarsal.

Kinematics analyses were performed on the same stepping sequences from which EMG activity was recorded and analyzed. Force data were calibrated and expressed in grams. EMG data were sampled at $2 \mathrm{kHz}$, filtered with a $20-500 \mathrm{~Hz}$ band-pass filter, and rectified. Briefly, the onset and offset of each EMG burst were marked to calculate burst duration. Mean EMG per burst was calculated by averaging the EMG amplitude within a burst. Integrated EMG (IEMG) was calculated as the product of the mean burst EMG amplitude and duration. To examine the modulation of EMG amplitudes between two muscles, joint probability distribution plots (Figure 10) were generated. These amplitudes were derived from a running five-point average of the mean amplitude of multiple normalized step cycles.

\section{STATISTICAL ANALYSES}

Statistically significant differences were determined using a oneway analysis of variance (ANOVA) using Monte Carlo simulations. The mean data from all the groups were pooled into a single data set before randomly sampling the data with replacement. Means from each group were used to estimate the $F$ value. This process

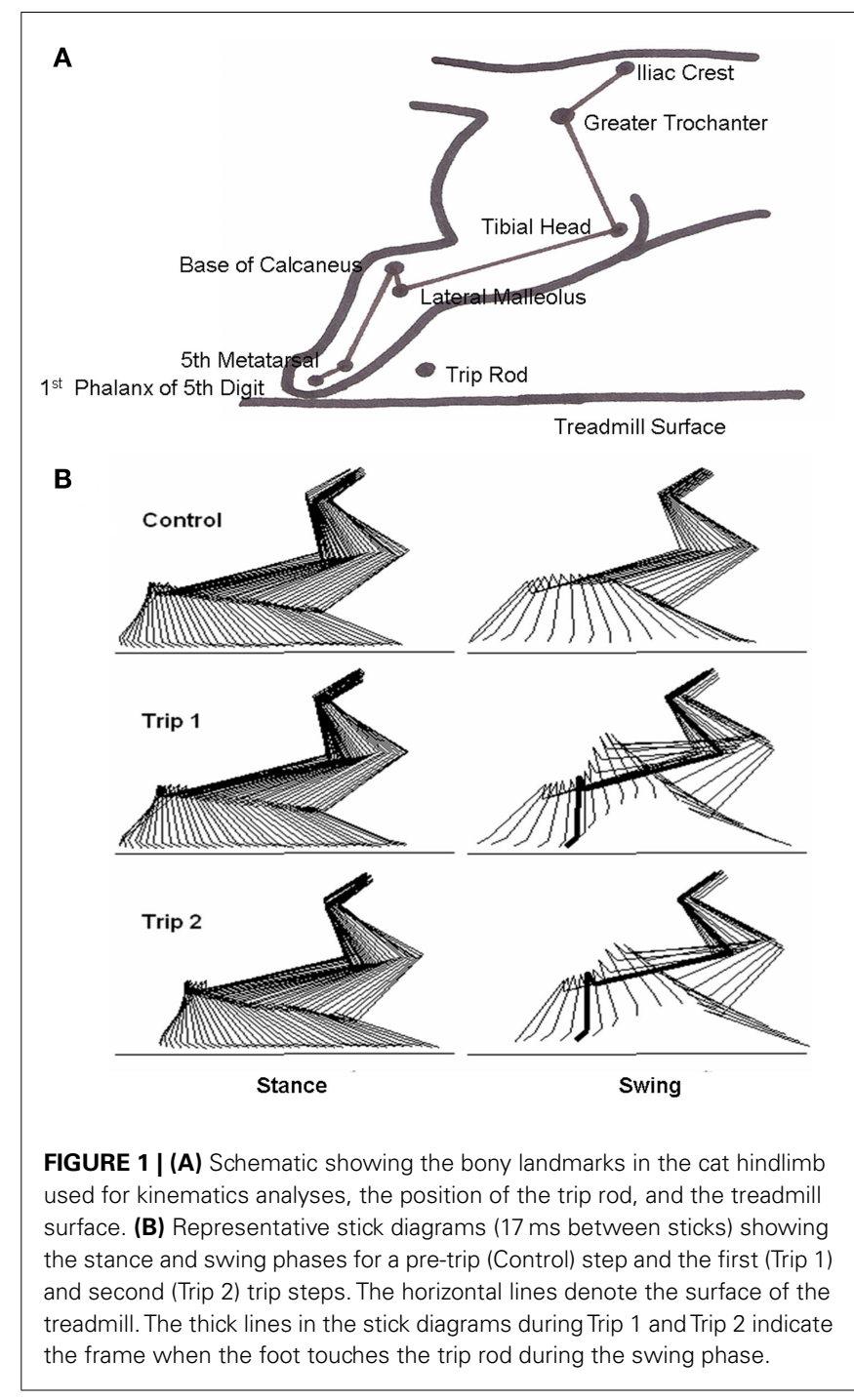

was repeated 10,000 times. The original $F$ value was compared to the simulated $F$ value to determine any overlap in the confidence bands. When the original $F$ value was outside the $95 \%$ confidence interval, the null hypothesis was rejected and there was a significant difference across groups at the $P<0.05$ level (Efron and Tibshirani, 1991).

\section{RESULTS}

\section{ADAPTATION IN JOINT KINEMATICS DURING THE TRIP RESPONSE}

Stick diagrams of the stance and swing phases for a control, first trip step (Trip 1), and second trip step (Trip 2) of a fivetrip step sequence, are shown in Figure 1B. For the control step, the hindlimb was allowed to proceed with an unperturbed swing phase. For Trip 1, the paw hit the trip rod, lifted over the rod, and extended forward further than the previous control step. For Trip 2 that immediately followed Trip 1, the trajectory of the paw changed prior to contact with the tripping rod, i.e., the paw changed its trajectory before it hit the trip rod. These observations were observed consistently during a number of sequences for the three cats analyzed. 


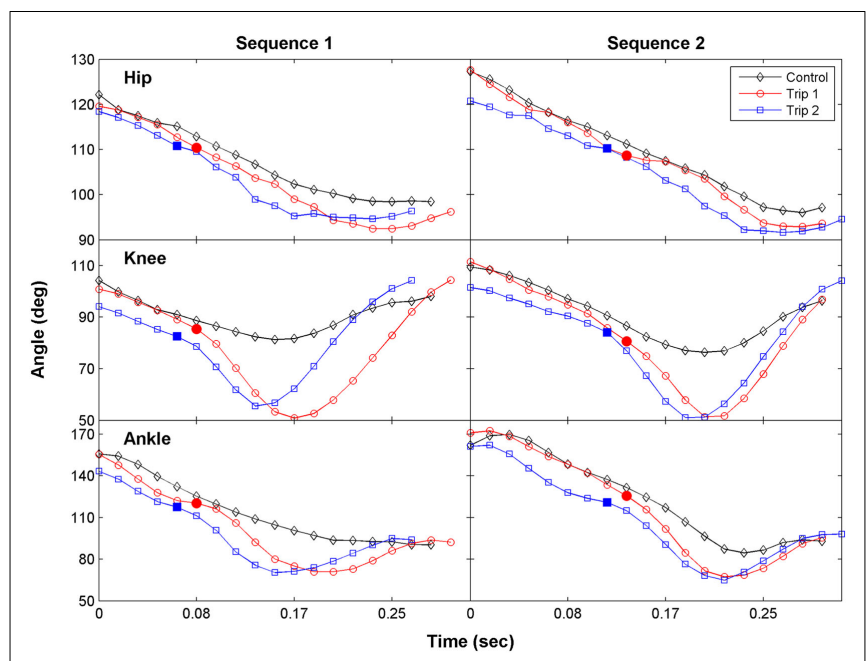

FIGURE 2 |The time course of the changes in the hip, knee, and ankle joint angles during the swing phase for a control, Trip 1, and Trip 2 step are shown for two tripping sequences in a representative cat. The solid symbols indicate the time when the foot touches the trip rod. The kinematics of Trip 2 reflect an adaptive response relative to Trip 1 at all three joints in both sequences. The stick figures in Figure 1B were generated from the data shown for the first sequence.

The hip, knee, and ankle joint angles for Trip 1 during swing were similar to the control step before contacting the trip rod, but each of these angles were smaller after contact (Figure 2, sequence 1). For Trip 2, the joint angles were smaller prior to the paw contacting the rod, indicating that the cat began to lift its hindlimb before contacting the rod. The magnitude of the change during the trip steps was greatest at the knee joint. Thus the increase in step height during the trip compared to the control steps was primarily attributable to an increase in knee flexion. Similar observations were made in a second sequence of the tripping experiment within that same session (Figure 2, sequence 2).

The trajectories of the knee, ankle, and foot (MTP joint) throughout the step cycle for the same steps shown in Figure 2 are shown in Figure 3. There was an earlier initiation of the swing phase in Trip 2 than Trip 1 in both sequences. These kinematics changes are shown for three tripping sequences in all three cats studied (Figure 4). The mean time when the foot started to lift prior to contact with the trip rod (Pre-contact time) was 150350 ms shorter for Trip 2 than Trip 1 in all three cats (Figure 4A). In addition, the distance from toe off to contact was shorter for Trip 2 than Trip 1 in all sequences in all three cats (Figure 4B). These data demonstrate a clear modification in the kinematics of the swing phase to allow the paw to be at a higher position when it reaches the point of the previous perturbation, thus increasing the likelihood for a successful completion of the swing phase.

\section{INCREASES IN MEAN STEP HEIGHT DURING THE RELEASE STEPS AFTER A SINGLE, SHORT, OR LONG BOUT OF TRIP STEPS}

Sequences with a varying number of trip steps resulted in an increase in the mean step height during the release steps compared to the control steps. For example, the mean step heights for the control (10 steps), long trip (60 steps), and release (10 steps)

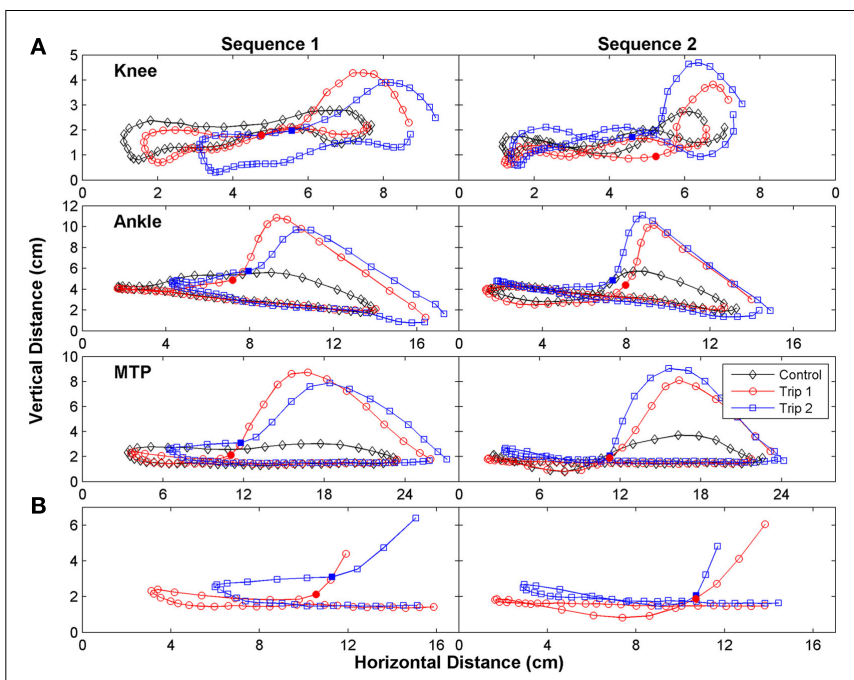

FIGURE 3 | The trajectory of the limb was changed immediately after the initial trip. (A) The trajectory of the knee (tibial head), ankle (lateral malleolus), and foot (metatarsophalangeal, MTP) for the same steps in Figure $\mathbf{2}$ are shown. (B) An expanded plot of the trajectory of the MTP during late stance and early swing of the Trip 1 and Trip 2 steps are depicted. The solid symbols in $(\mathbf{A}, \mathbf{B})$ indicate when the foot touches the trip rod. Note the earlier initiation of the swing phase in Trip 2 than Trip 1 for both sequences.

steps during a single sequence were $2.8,5.5$, and $4.8 \mathrm{~cm}$, respectively (Figure 5A). In another cat, the mean step heights for the control ( 9 steps), shorter trip series (10 steps), and release ( 9 steps) steps during a single sequence were $2.1,4.9$, and $2.8 \mathrm{~cm}$, respectively (Figure 5B). In fact, the mean step height was significantly higher during the release steps compared to the control steps after a single trip sequence (ranging from 10 to 60 steps) in all four cats studied. In another example, a trip sequence of only two steps resulted in an increase in step height from $1.5 \mathrm{~cm}$ for the control steps (10 steps) to $2.5 \mathrm{~cm}$ during the release steps (9 steps; Figure 5C). This elevated step height for the release steps occurred in spite of the fact that the hindlimb collapsed in the first release step (this step was excluded when calculating step height). The subsequent series of 10 trip steps had no further effect on mean step height during the ensuing release steps, but the mean step height still was elevated compared to the control steps.

To further determine the temporal features of the tripping stimulus, a perturbation was imposed on every third step (Figure 5D). The sequence involved 9 control steps, a total of 10 trip steps with 2 release steps in between each perturbation, and a final 9 release steps. In every case the height of the two release steps between each trip step was elevated above control. In addition, the mean step height for the final nine release steps was higher than for the control steps, i.e., 3.2 vs. $2.5 \mathrm{~cm}$. Occasionally the cats adapted the height of the swing phase sufficiently to step over the rod without touching it. For example, as shown in Figure 6 the cat's paw cleared the bar at steps $14,16,30$, and 35 in a series of 52 tripped steps. All of these data demonstrate that the spinal cats adjusted the step trajectory as early as in the second trip step to lift the leg higher. 

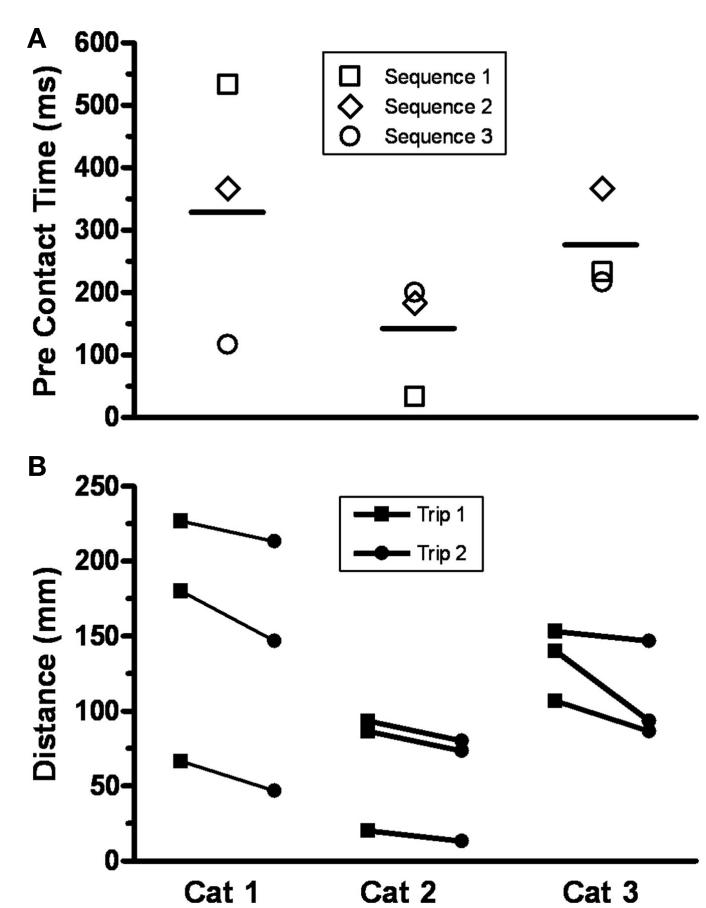

FIGURE 4 | (A) The pre-contact time, defined as the time when the foot started to lift prior to contact with the trip rod, for Trip 2 compared to Trip 1 for three sequences is shown for three cats. The horizontal bar is the mean for three sequences for each cat (range, 150-350 ms). A shorter pre-contact time for Trip 2 than Trip 1 was present in all nine cases. (B) The distance from toe off to contact with the trip rod for Trip 1 and Trip 2 for three sequences is shown for the same three cats. All distances were shorter for Trip 2 than Trip 1.

\section{IMPACT FORCE ON THE TRIP ROD DECREASES WITH CONSECUTIVE SEOUENCES}

In general, the impact force decreased with repeated sequences. For example, during the performance of three sequences the mean impact force progressively decreased from $554 \mathrm{~g}$ (range, 282-644 g) during the first sequence, to $319 \mathrm{~g}$ (range, 202-512 g) for the second sequence, to $247 \mathrm{~g}$ (range, 173-302 g) for the third sequence (Figure 7A). Note that the average step height during the release steps after each series of trip steps was higher than during the control steps. The ratio of impact force:step height (Figure 7B) showed the same pattern as for impact force (Figure 7A). This decrease in the ratio across trip sequences was, at least in part, due to a lower horizontal velocity during the swing prior to contact, i.e., $0.75,0.63$, and $0.57 \mathrm{~m} / \mathrm{s}$ for in first, second, and third tripping sequence. A similar pattern was observed in three cats. Combined, these data indicate that the spinal sensorimotor circuitry made kinematics adjustments to minimize the impact force as early as the second trip step.

\section{MODULATION IN THE EMG PATTERNS DURING TRIP-RELEASE SEOUENCES}

EMG analysis was performed on four hindlimb muscles (St, TA, Sol, and MG) of the tripped hindlimb in two cats and on two muscles (St and TA) in a third cat. The mean EMG burst durations,

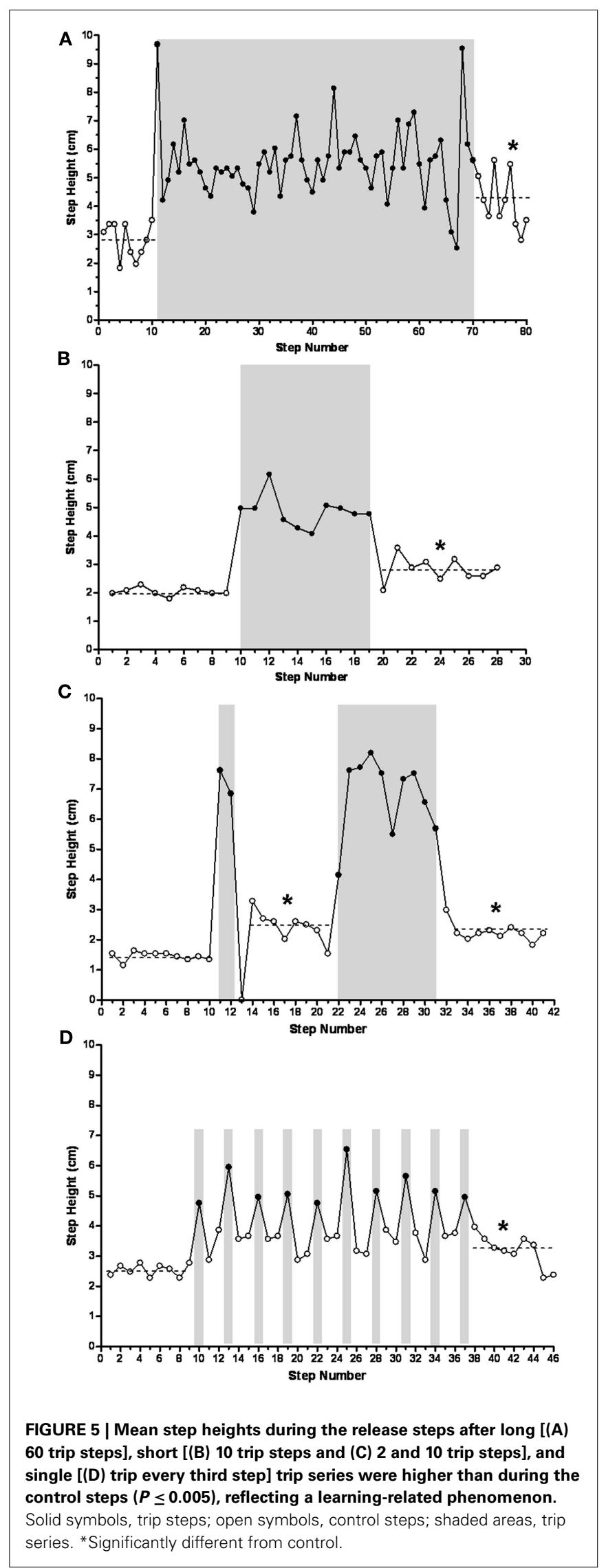




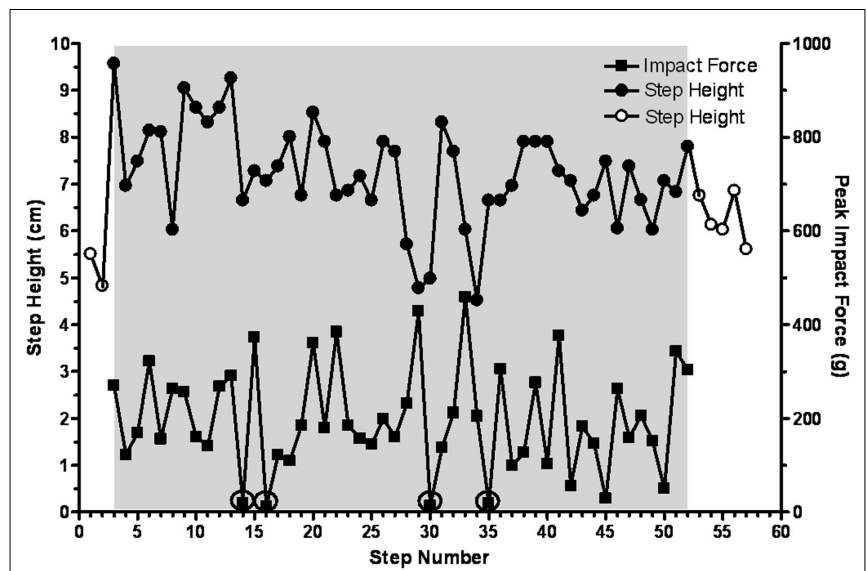

FIGURE 6 | Step height and peak impact force on the trip rod are shown for $\mathbf{5 2}$ consecutive trip steps for a representative cat. Four steps during which the limb avoided and cleared the trip rod are circled. Solid symbols, trip steps; open symbols, control steps; shaded area, trip series.

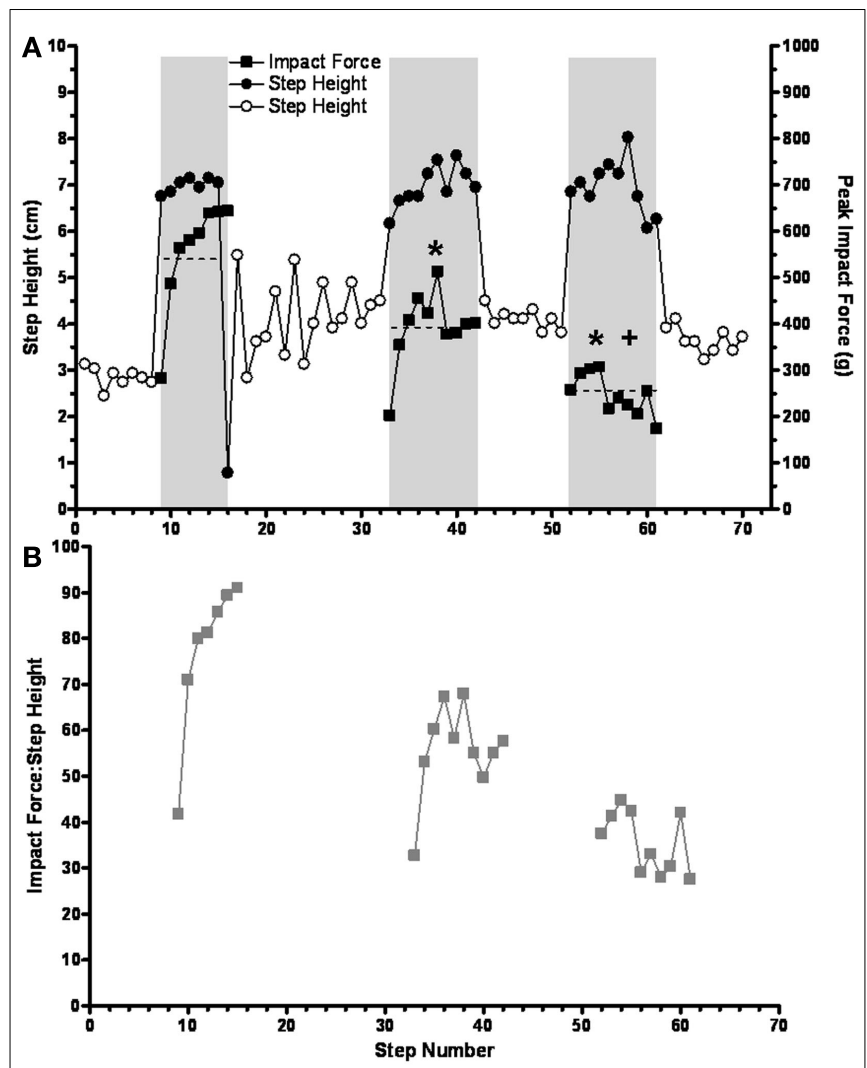

FIGURE 7 | (A) The mean impact force on the trip rod (horizontal dashed lines) is shown to progressively decrease during three successive short trip-release sequences. Solid symbols, trip steps; open symbols, control steps; shaded areas, trip series. (B) The ratios of peak impact force and step height are shown for the same trip sequences in (A). Note that the results in both $(\mathbf{A})$ and $(\mathbf{B})$ reflect a learning-related phenomenon that occurs over a time frame of seconds. ${ }^{*}$ and + Significantly different from the first and second trip sequence, respectively.

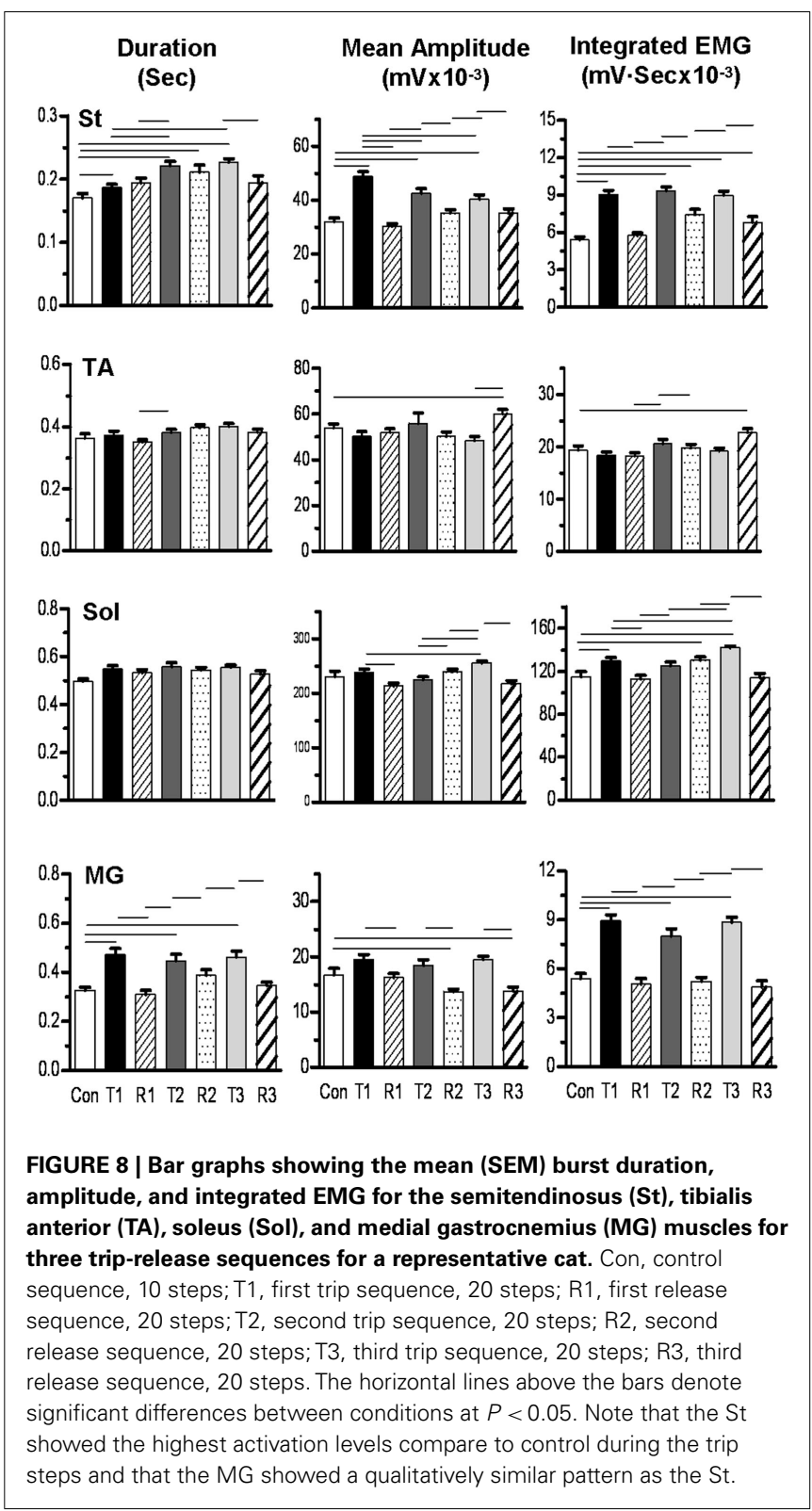

EMG burst amplitudes, and IEMGs for a control (10 steps), three trip (20 steps per series), and three release (20 steps per series) sequences for one cat are shown in Figure 8. In general, the mean burst durations for the St and MG, but not the Sol and TA, were longer for the trip compared to control steps. The mean EMG burst durations for the release steps generally were shorter than for the trip steps within a sequence for the MG, with no apparent trend for all other muscles. Mean EMG amplitudes and IEMG in the St, MG, and Sol were generally lower for the release steps compared to the trip steps. The other two cats in which EMG was determined showed similar activation patterns.

To look at the changes in the timing of the EMG, rectified raw EMG for the control, trip 1, release 1, and trip 2 sequences shown in Figure $\mathbf{8}$ are plotted in Figure 9. For the St the amplitude at the end of the EMG burst was markedly higher $(P<0.05)$ for the 
first trip series than the control series (Figure 9A). For the second trip sequence (Trip 2), the pattern of the St EMG was similar to control (Figure 9B). For the first trip sequence, the TA appears to be activated earlier and to have an EMG burst amplitude similar to control. The EMG amplitudes of the extensor muscles (Sol and MG) were higher $(P<0.05)$ in the beginning of the stance phase for the trip compared to the control and release sequences (see ${ }^{*}$ in Figure 9A). The levels of activation of the TA, Sol, and MG vs. the St (the muscle showing the most consistent changes in response to the tripping stimulus) for a sequence of 10 control, 20 trip, and 20 release steps are shown in Figure 10. Note that the plots for the control and release steps are similar (Figures 10A,C), whereas the plots for the trip steps show an elevated level of activation for the St and a higher level of co-activation for the St with the TA (Figure 10B). The temporal sequence of the modulation of the St EMG amplitude immediately after impact on the rod is shown by the line connecting consecutive data points (green symbols) in Figure 10B. Note that the elevated St activity and the co-activation between the TA and St during the tripping steps had no impact on the clear reciprocal relationship between the St with either the Sol or MG. Similar observations were made in two other cats.

\section{DISCUSSION}

The "stumbling response" of the hindlimbs in complete, lowthoracic spinal cats was characterized more than three decades ago (Forssberg, 1979). These experiments demonstrated that a single instantaneous perturbation of a complex motor task, i.e., stepping, would result in a successful and instantaneously newly adopted neural control strategy, at least for the duration of the ipsilateral swing and contralateral stance phase of the step cycle for the whole hindquarters to sustain continuous stepping. If the same mechanical or electrical stimulus was applied during the stance phase of the step cycle of the spinal cat, there was an immediate hyperextension of the ipsilateral limb, indicating a "state dependent" feature of spinal "decision making." There, however, has been no systematic examination as to whether there are residual effects manifested in subsequent step cycles in complete spinal animals.

McVea and Pearson (2007) reported an elevated height of the swing phase after 20 or more consecutive perturbations of the swing phase in normal cats. This change in swing trajectory persisted up to $24 \mathrm{~h}$ and the magnitude of this effect generally reached a plateau within 120 repetitions of the perturbation. This effect was not clearly evident after decerebration, and the authors concluded that this phenomenon was mediated by supraspinal descending systems. There were, however, some short-term residual effects of repetitive stumbling in the decerebrated cats, but the elevated height of the swing phase was sustained for only about five to seven steps after removal of the perturbation. Given the multiple observations that have shown learning-related phenomena in the performance of a motor task within the lumbosacral spinal circuitry with time courses of the learned tasks ranging from minutes to weeks (Edgerton et al., 2001b; de Leon et al., 2002; Timoszyk et al., 2002; Liu et al., 2005), we examined the persistence of the tripping response in adult, chronic spinal cats that had been trained to step. In these experiments we examined the time frame of seconds over which experience-dependent modulation of the control of stepping can occur in the spinal circuitry without any supraspinal influence.

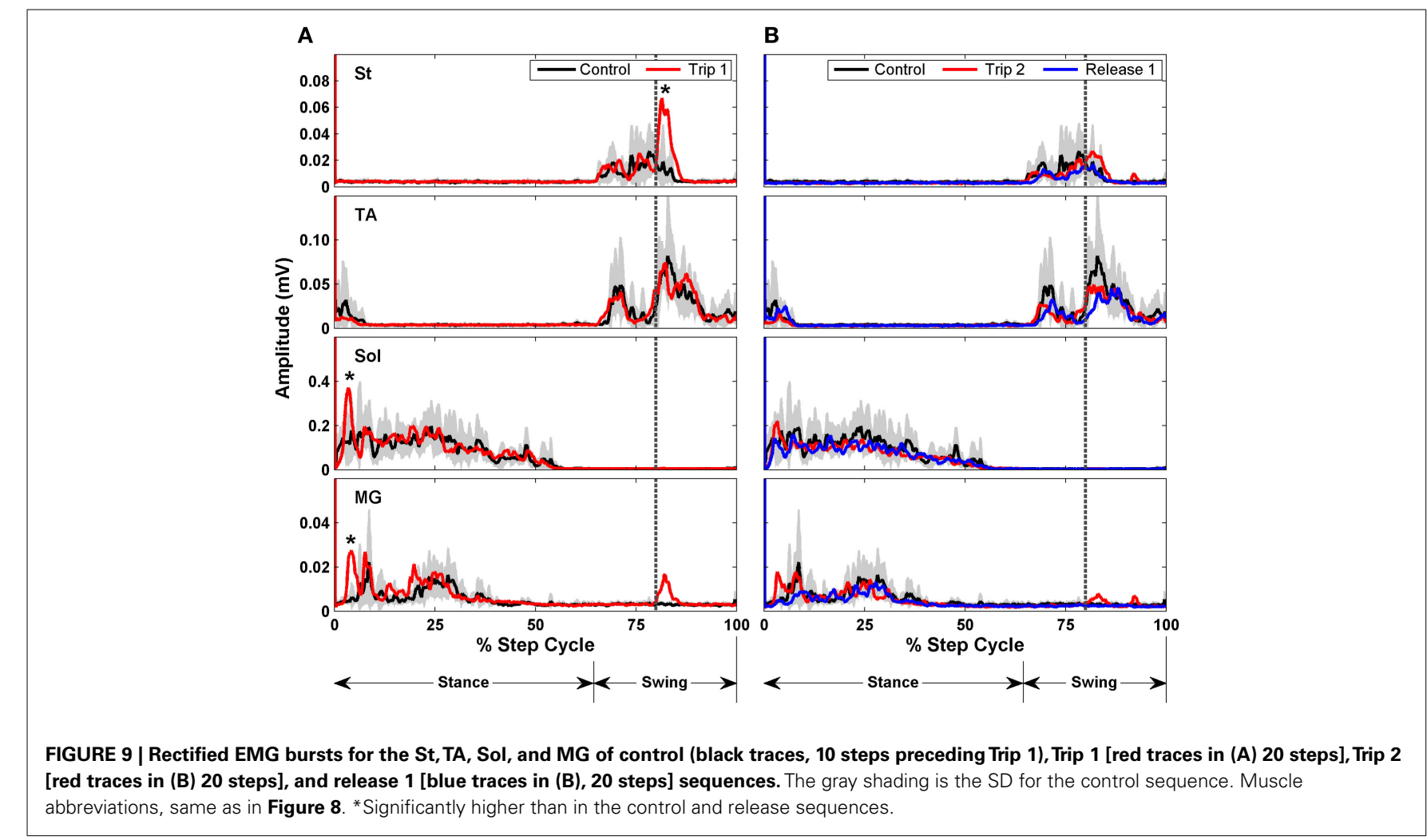




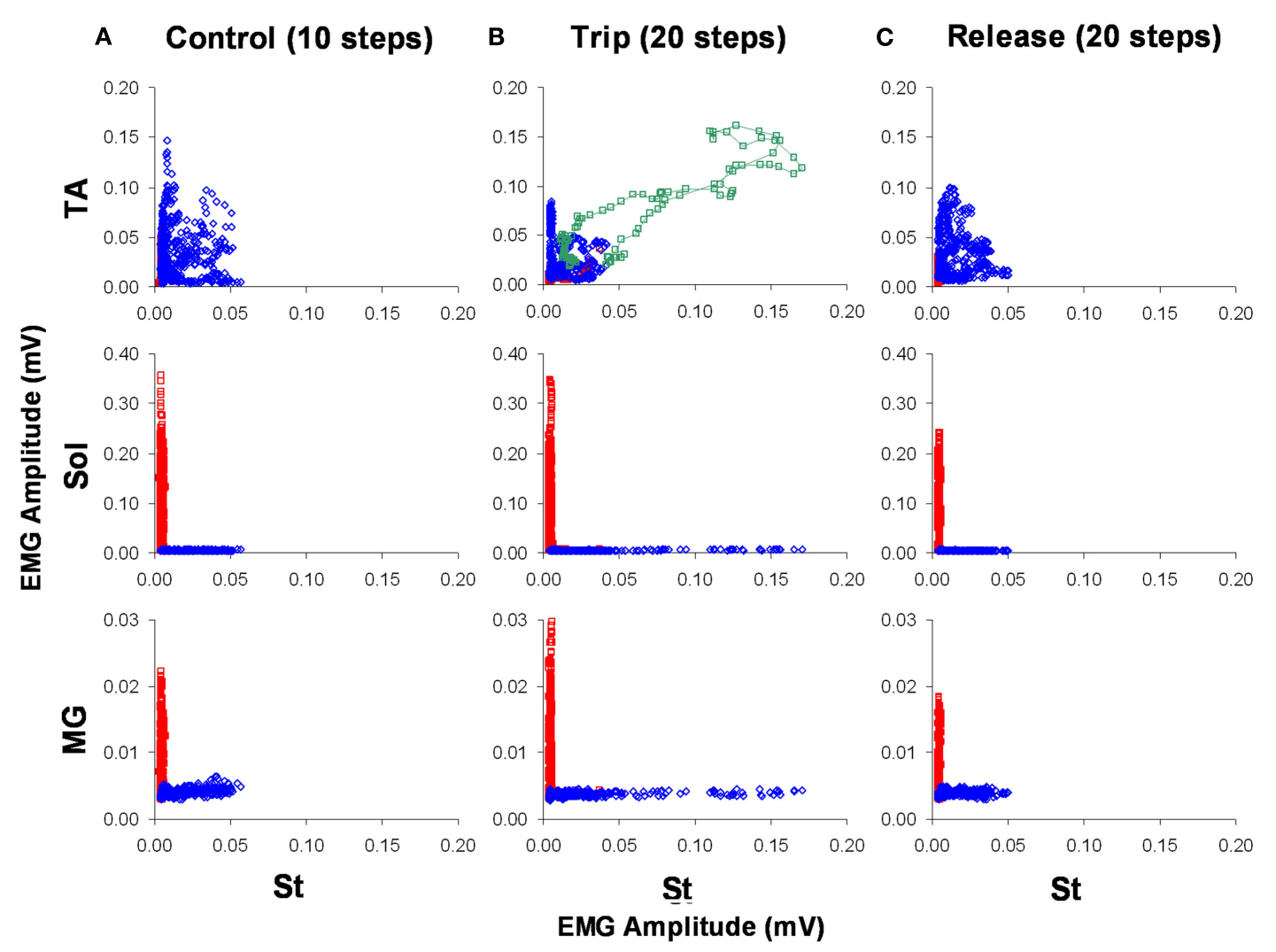

FIGURE 10 | The level of activation of the TA, Sol, and MG vs. the St for a sequence of 10 control (A), 20 trip (B), and 20 release steps (C) is shown. Blue and red represent the swing and stance phases of the step cycle, respectively. The green symbols in (B) represent the co-activation between the TA and St immediately after the trip. The data points are a five-point running average of the mean normalized EMG. Note the higher level of activation of the St during the trip compared to the control and release steps.
The present data demonstrate adaptive responses to a perturbation of the kinematics of the hindlimb in complete spinal cats occurring within a single step cycle as well as over as many as 60 continuous step cycles. These adaptive responses enhance the probability of sustaining successful stepping while being challenged with a specific mechanical perturbation by elevating the hindlimbs in a manner that tends to avoid the perturbation. From a more highly integrative and conceptual perspective, these responses reflect "recognition" of a specific complex afferent pattern induced by the stimulus that results in an adjustment to a subsequent encounter within the time frame of a single step cycle, i.e., within the same step cycle the trajectory of the paw is changed in a way that tends to avoid the previous perturbation (Figures 1-4). The neuromotor strategy that mediates this ipsilateral hyperflexion during the swing phase after the tripping stimulus seems to be rather consistent for different experimental preparations ranging from intact locomotion to fictive locomotion (Quevedo et al., 2005; McVea and Pearson, 2007). A common feature of this response is an increase in step height and earlier initiation of activation, particularly of motor pools associated with knee flexion. In the present study these were the most predominant and consistent changes observed in response to the tripping stimulus.
In essence the immediate response (within the same step cycle as the initial perturbation) demonstrates a feed-forward control mechanism within the spinal circuitry, i.e., a single instantaneous sensory event that interrupts a step can be recognized as such, and a response is initiated which modifies the limb trajectory not only to complete that swing phase, but also to adjust the neural control needed to avoid or minimize the perturbation in the succeeding step cycle. The initial response in modifying the limb trajectory within a single step cycle would seem to reflect "evolutionary learning" (Edgerton et al., 2001a) in that a similar ability to adjust the kinematics of a limb to a continuously changing environment apparently has been acquired in a wide range of invertebrates and vertebrates (Grillner, 1981). The more sustained responses that occur as a result of repetitive presentations of the perturbations, however, could be attributed to more classical learning phenomena occurring over a range of time frames, undoubtedly engaging different mechanisms.

Much of the emphasis on the response to stumbling during locomotion has been on the initial "reflex" response with less attention given to the responses that are more delayed and sustained over multiple step cycles. These latter responses are highly coordinated responses requiring larger scale circuitries to control those motor pools that generate locomotion. Early and late responses to 
stumbling perturbations that can sustain successful stepping after a stumble during locomotion have been observed in uninjured human adults (Schillings et al., 2000) and infants (Pang et al., 2003), decerebrated ferrets (Lou and Bloedel, 1988), intact cats (McVea and Pearson, 2007), and chronic spinal cats (Forssberg et al., 1975). Other more complex perturbations, such as applying force fields at specific phases of the step cycle in spinal rats, also demonstrate that there is a range of highly coordinated and immediate as well as delayed motor responses that have memory-related properties (Timoszyk et al., 2002; Heng and de Leon, 2007). The combination of the studies noted above demonstrate that the locomotor spinal circuitry can recognize, predict, and adapt to brief, instantaneous as well as more prolonged perturbations of complex motor tasks such as stepping.

Other examples demonstrate the detailed control that can be exhibited by the proprioceptive input to the spinal cord in complete spinal animals. Spinal rats and cats have the ability to step in different directions when stepping on a treadmill (Grillner, 1981; Heng and de Leon, 2007; Courtine et al., 2009). Decerebrate (Musienko et al., 2012) and spinal (unpublished observations) cats can make corrective responses to proprioceptive input (lateral displacement of the hindquarters) to maintain balance during stepping. Similar responses in balance control during standing have been reported in a human subject after complete paralysis (Harkema et al., 2011). In addition, Wernig and Muller (1991) reported that a completely paralyzed individual could initiate stepping by shifting his body weight, stretching the hip, and leaning forward. Each of these observations, in addition to the present data, demonstrate a significant level of sensory control that goes well beyond the control of the more stereotypical unperturbed stepping that has been demonstrated in chronic spinal cats. These observations reflect more than simple reflexdriven responses as they encompass a more sophisticated processing of sensory input, including feedforward as well as feedback mechanisms.

\section{REFERENCES}

Barbeau, H., and Rossignol, S. (1987). Recovery of locomotion after chronic spinalization in the adult cat. Brain Res. 412, 84-95.

Choi, J. T., and Bastian, A. J. (2007). Adaptation reveals independent control networks for human walking. Nat. Neurosci. 10, 1055-1062.

Chopin, S. F., and Buerger, A. A. (1976). Instrumental avoidance conditioning in the spinal rat. Brain Res. Bull. 1, 177-183.

Courtine, G., Gerasimenko, Y., van den Brand, R., Yew, A., Musienko, P., Zhong, H., Song, B., Ao, Y., Ichiyama, R. M., Lavrov, I., Roy, R. R., Sofroniew, M. V., and Edgerton, V. R. (2009). Transformation of nonfunctional spinal circuits into functional states after the loss of brain input. Nat. Neurosci. 12, 1333-1342.

de Leon, R. D., Hodgson, J. A., Roy, R. R., and Edgerton, V. R.

The present results may be interpreted as a reflection of central pattern generation. Central pattern generation, however, is cyclic activity that occurs without any phasic sensory input. The spinal model used herein, in contrast, encompasses the ability of the spinal central pattern generators to dynamically process complex sensory ensembles, make detailed decisions, in real time, and execute the appropriate motor output (Courtine et al., 2009). In fact, the spinal cord circuitry recognizes and habituates (learns) to the sensory input, facilitating the avoidance of an obstacle during stepping. Thus the present results should be interpreted within a context that exceeds what is routinely considered to be central pattern generation.

In summary, the spinal locomotor circuitry can recognize and translate a simple, instantaneous stimulus (perturbation) into a "useful" adaptation of a complex motor task (e.g., stepping). This ability to sustain such an adaptive strategy demonstrates the degree to which the spinal locomotor circuitry accommodates in a rather routine way to continuing changes in environmental events, providing a neural substrate for making these behaviors essentially "automatic," i.e., requiring little or no supraspinal intervention. This capability is clearly evident in normal human adults and infants and decerebrate cats and some features of these immediate and later responses have been observed during fictive locomotion in the cat (Quevedo et al., 2005). The present data, combined with other studies addressing longer time frames (Liu et al., 2005), demonstrate that a wide range of accommodating responses covering a time frame ranging from milliseconds to minutes can be generated and that these responses also can be "remembered" over this time frame in a manner that facilitates sustained locomotion within the spinal locomotor circuitry.

\section{ACKNOWLEDGMENTS}

This work was funded by National Institute of Health Grant NS 16333.

Trauma: Regeneration, Neural Repair and Functional Recovery: Progress in brain research, Vol. 137, eds L. McKerracher, G. Doucet, and S. Rossignol (Netherlands: Elsevier Science BV), 141-149.

de Leon, R. D., Tamaki, H., Hodgson, J. A., Roy, R. R., and Edgerton, V. R. (1999). Hindlimb locomotor and postural training modulates glycinergic inhibition in the spinal cord of the adult spinal cat. J. Neurophysiol. 82, 359-369.

Di Giorgio, A. M., and Menzio, P. (1946). Comportamento degli arti posteriori dopo deafferntazione e lesione unilaterale del cervelletto. Boll. Soc. Ital. Biol. Sper. 22, 827-828.

Edgerton, V. R., de Leon, R. D., Harkema, S. J., Hodgson, J. A., London, N., Reinkensmeyer, D. J., Roy, R. R., Talmadge, R. J., Tillakaratne, N. J., Timoszyk, W., and Tobin, A. (2001a). Topical review: retraining the injured spinal cord. J. Physiol. 533, 15-22.

Edgerton, V. R., Roy, R. R., and de Leon, R. D. (2001b). "Neural Darwinism in the mammalian spinal cord," in Spinal Cord Plasticity: Alterations in Reflex Function, eds M. M. Patterson and J. W. Grau (Boston: Kluwer Academic Publishers), 185-206.

Efron, B., and Tibshirani, R. (1991). Statistical analysis in the computer age. Science 253, 390-395.

Forssberg, H. (1979). Stumbling corrective reaction: a phase-dependent compensatory reaction during locomotion. J. Neurophysiol. 42, 936-953.

Forssberg, H., Grillner, S., and Rossignol, S. (1975). Phase dependent reflex reversal during walking in chronic spinal cats. Brain Res. 85, 103-107. 
Grillner, S. (1981). "Control of locomotion in bipeds, tetrapods, and fish," in Handbook of Physiology. The Nervous System. Motor Control, eds M. Brookhart and V. B. Mountcastle (Bethesda, MD: American Physiological Society), 1179-1236.

Harkema, S., Gerasimenko, Y., Hodes, J., Burdick, J., Angeli, C., Chen, Y., Ferreira, C., Willhite, A., Rejc, E., Grossman, R. G., and Edgerton, V. R. (2011). Effect of epidural stimulation of the lumbosacral spinal cord on voluntary movement, standing, and assisted stepping after motor complete paraplegia: a case study. Lancet 377 , 1938-1947.

Heng, C., and de Leon, R. D. (2007). The rodent lumbar spinal cord learns to correct errors in hindlimb coordination caused by viscous force perturbations during stepping. J. Neurosci. 27, 8558-8562.

Liu, C., Ziegler, M. D., Udwadia, Z. F., Khristy, W., Guu, J. J., Ali, N. J., Ahn, S. N., Deleon, R. D., Meliklan, R., Roy, R. R., Zhong, H., Tobin, A. J., Edgerton, V. R., and Tillakaratne, N. J. K. (2005). Short-Term and LongTerm Spinal Learning: Effects of Protein Synthesis Inhibition, Program
No. 396.10. Washington, DC: Society for Neuroscience.

Lou, J. S., and Bloedel, J. R. (1988). A new conditioning paradigm: conditioned limb movements in locomoting decerebrate ferrets. Neurosci. Lett. 84, 185-190.

Lovely, R. G., Gregor, R. J., Roy, R. R., and Edgerton, V. R. (1990). Weight-bearing hindlimb stepping in treadmill-exercised adult spinal cats. Brain Res. 514, 206-218.

McVea, D. A., and Pearson, K. G. (2007). Long-lasting, contextdependent modification of stepping in the cat after repeated stumblingcorrective responses. J. Neurophysiol. 97, 659-669.

Musienko, P., Courtine, G., and Tibbs, J. E., Kilimnik, V., Savochin, A., Garfinkel, A., Roy, R. R., Edgerton, V. R., and Gerasimenko, Y. (2012). Somatosensory control of balance during locomotion in decerebrated cat. J. Neurophysiol. 107, 2071-2082.

Pang, M. Y., Lam, T., and Yang, J. F. (2003). Infants adapt their stepping to repeated trip-inducing stimuli. $J$. Neurophysiol. 90, 2731-2740.

Pierotti, D. J., Roy, R. R., Gregor, R. J., and Edgerton, V. R. (1989). Electromyographic activity of cat hindlimb flexors and extensors during locomotion at varying speeds and inclines. Brain Res. 481, 57-66.

Quevedo, J., and Stecina, K., Gosgnach, S., and McCrea, D. A. (2005). Stumbling corrective reaction during fictive locomotion in the cat. J. Neurophysiol. 94, 2045-2052.

Roy, R. R., Hodgson, J. A., Lauretz, S. D., Pierotti, D. J., Gayek, R. J., and Edgerton, V. R. (1992). Chronic spinal cord-injured cats: surgical procedures and management. Lab. Anim. Sci. 42, 335-343.

Schillings, A. M., Van Wezel, B. M. Mulder, T., and Duysens, J. (2000). Muscular responses and movement strategies during stumbling over obstacles. J. Neurophysiol. 83, 2093-2102.

Timoszyk, W. K., de Leon, R. D., London, N., Roy, R. R., Edgerton, V. R., and Reinkensmeyer, D. J. (2002). The rat lumbosacral spinal cord adapts to robotic loading applied during stance. J. Neurophysiol. 88, 3108-3117.

Wernig, A., and Muller, S. (1991). "Improvement of walking in spinal cord injured persons after treadmill training," in Restorative Neurology, ed. A. Wernig
(Amsterdam: Elsevier Science BV), 475-485.

Conflict of Interest Statement: The authors declare that the research was conducted in the absence of any commercial or financial relationships that could be construed as a potential conflict of interest.

Received: 01 March 2012; paper pending published: 15 March 2012; accepted: 04 April 2012; published online: 01 May 2012.

Citation: Zhong H, Roy RR, Nakada KK, Zdunowski S, Khalili N, de Leon $R D$ and Edgerton VR (2012) Accommodation of the spinal cat to a tripping perturbation. Front. Physio. 3:112. doi: 10.3389/fphys.2012.00112

This article was submitted to Frontiers in Integrative Physiology, a specialty of Frontiers in Physiology.

Copyright (C) 2012 Zhong, Roy, Nakada, Zdunowski, Khalili, de Leon and Edgerton. This is an open-access article distributed under the terms of the Creative Commons Attribution Non Commercial License, which permits non-commercial use, distribution, and reproduction in other forums, provided the original authors and source are credited. 